University of Nebraska - Lincoln

DigitalCommons@University of Nebraska - Lincoln

Publications from USDA-ARS / UNL Faculty

U.S. Department of Agriculture: Agricultural

Research Service, Lincoln, Nebraska

2015

\title{
Simultaneous conversion of all cell wall components by an oleaginous fungus without chemi-physical pretreatment
}

\author{
Shangxian Xie \\ Texas A\&M University \\ Xing Qin \\ Texas A\&M University \\ Yanbing Cheng \\ Texas A\&M University \\ Dhrubojyoti Laskar \\ Washington State University \\ Weichuan Qiao \\ Texas A\&M University \\ See next page for additional authors
}

Follow this and additional works at: https://digitalcommons.unl.edu/usdaarsfacpub

Xie, Shangxian; Qin, Xing; Cheng, Yanbing; Laskar, Dhrubojyoti; Qiao, Weichuan; Sun, Su; Reyes, Luis H.; Wang, Xin; Dai, Susie Y.; Sattler, Scott E.; Kao, Katy; Yang, Bin; Zhang, Xiaoyu; and Yuan, Joshua S., "Simultaneous conversion of all cell wall components by an oleaginous fungus without chemi-physical pretreatment" (2015). Publications from USDA-ARS / UNL Faculty. 1861.

https://digitalcommons.unl.edu/usdaarsfacpub/1861

This Article is brought to you for free and open access by the U.S. Department of Agriculture: Agricultural Research Service, Lincoln, Nebraska at DigitalCommons@University of Nebraska - Lincoln. It has been accepted for inclusion in Publications from USDA-ARS / UNL Faculty by an authorized administrator of DigitalCommons@University of Nebraska - Lincoln. 


\section{Authors}

Shangxian Xie, Xing Qin, Yanbing Cheng, Dhrubojyoti Laskar, Weichuan Qiao, Su Sun, Luis H. Reyes, Xin Wang, Susie Y. Dai, Scott E. Sattler, Katy Kao, Bin Yang, Xiaoyu Zhang, and Joshua S. Yuan 


\section{Green Chemistry}

\section{PAPER}

CrossMark

\&lick for updates

Cite this: Green Chem., 2015, 17, 1657

\section{Simultaneous conversion of all cell wall components by an oleaginous fungus without chemi-physical pretreatment $\uparrow$}

\author{
Shangxian Xie, ${ }^{a, b}$ Xing Qin, ${ }^{b}$ Yanbing Cheng, ${ }^{b}$ Dhrubojyoti Laskar, ${ }^{c}$ Weichuan Qiao, ${ }^{b}$ \\ Su Sun, ${ }^{b}$ Luis H. Reyes, ${ }^{d}$ Xin Wang, ${ }^{b}$ Susie Y. Dai, ${ }^{e}$ Scott E. Sattler, ${ }^{f}$ Katy Kao, ${ }^{d}$ \\ Bin Yang, ${ }^{c}$ Xiaoyu Zhang*a and Joshua S. Yuan*a,b
}

\begin{abstract}
Lignin utilization during biomass conversion has been a major challenge for lignocellulosic biofuel. In particular, the conversion of lignin along with carbohydrate for fungible fuels and chemicals will both improve the overall carbon efficiency and reduce the need for chemical pretreatments. However, few biomass-converting microorganisms have the capacity to degrade all cell wall components including lignin, cellulose, and hemicellulose. We hereby evaluated a unique oleaginous fungus strain, Cunninghamella echinulata FR3, for its capacity to degrade lignin during biomass conversion to lipid, and the potential to carry out consolidated fermentation without chemical pretreatment, especially when combined with sorghum (Sorghum bicolor) bmr mutants with reduced lignin content. The study clearly showed that lignin was consumed together with carbohydrate during biomass conversion for all sorghum samples, which indicates that this organism has the potential for biomass conversion without chemical pretreatment. Even though dilute acid pretreatment of biomass resulted in more weight loss during fungal fermentation than untreated biomass, the lipid yields were comparable for untreated $\mathrm{bmr} 6 / \mathrm{bmr} 12$ double mutant and dilute acid-pretreated wild-type biomass samples. The mechanisms for lignin degradation in oleaginous fungi were further elucidated through transcriptomics and chemical analysis. The studies showed that in C. echinulata FR3, the Fenton reaction may play an important role in lignin degradation. This discovery is among the first to show that a mechanism for lignin degradation similar to those found in white and brown rot basidiomycetous fungi exists in an oleaginous fungus. This study suggests that oleaginous fungi such as $C$. echinulata FR3 can be employed for complete biomass utilization in a consolidated platform without chemical pretreatment or can be used to convert lignin waste into lipids.
\end{abstract}

Received 8th August 2014, Accepted 1st December 2014 DOI: $10.1039 / c 4 g c 01529 k$ www.rsc.org/greenchem conversion follows three steps, namely pretreatment, saccharification, and fermentation ${ }^{1,2}$ A major goal of pretreatment is to disrupt the lignin structure to make cellulose more accessible to saccharification. ${ }^{1,3}$ However, most of the chemical or physical pretreatment strategies require high energy input and harsh conditions such as high temperature and/or extreme $\mathrm{pH}$, which often also result in toxic waste. Biological pretreatment, on the other hand, can be carried out under milder conditions and be more environmentally friendly. However, it is still challenging to apply biological pretreatment for biofuel production due to the long reaction time. Lignin modification on feedstock thus emerged as an alternative strategy for reducing recalcitrance and developing cost-effective bioconversion with simplified pretreatment. ${ }^{4,5}$

Traditionally, lignin modification was extensively studied in model plants such as Medicago, Arabidopsis, and Nicotiana. ${ }^{4,6,7}$ Recently, significant advances have been made in the improvement of grasses, including sorghum and switchgrass, as biofuel feedstocks. ${ }^{8-10}$ However, most research on the lignin 
modification of grasses has been carried out via genetic modification, which creates regulatory issues for commercial implementation. In contrast, a unique set of chemically mutagenized lignin modification brown midrib (bmr) mutants was developed in sorghum as a major asset in determining how modifications to the lignin biosynthesis pathway impact biomass conversion. ${ }^{11,12}$ The biomass from bmr6 or bmr12 plants was significantly reduced in lignin content and altered in lignin composition relative to wild-type biomass. Each mutant showed increased saccharification and ethanol conversion efficiency for cellulose. ${ }^{13}$ When combined with a double mutant, the effects were additive in reducing the lignin content and increasing the conversion efficiency. ${ }^{14}$ Despite the previous advances in ethanol platforms, few studies focused on how lignin modification will impact the oleaginous fungus conversion of biomass into lipid. Also unknown was whether feedstock lignin modification could promote the $C$. echinulata FR3 conversion of biomass without chemi-physical pretreatment.

Recently, lipid production from biomass using oleaginous microbes has been explored. ${ }^{15,16}$ Many studies have reported that oleaginous fungi, including Mortierella isabellina and Mucor circinelloides, could produce lipid from lignocellulolytic hydrolysates. ${ }^{15,16}$ Recent research studies also showed that oleaginous bacteria, such as Rhodococcus opacus PD630, may have the potential capacity to use lignin as the sole carbon resource for lipid production. ${ }^{17}$ In addition, oleaginous yeast can also be used for converting biomass hydrolysates. ${ }^{18}$ Even though the overall conversion rate of carbohydrates to lipid is much lower than that of ethanol, the oleaginous fungus-based platform has at least three major advantages. First, from a broader biodiesel industry perspective, lignocellulosic biomass provides a reliable feedstock for the significant amount of lipid needed for biodiesel refineries. Second and more importantly, oleaginous fungal species have been widely used for the biotransformation of aromatic compounds, suggesting the possibility of using these species to convert lignin along with carbohydrates into biofuel products. ${ }^{19,20}$ For example, we identified a fast-growing oleaginous fungus, C. echinulata FR3, with the capacity to accumulate lipids at more than $40 \%$ of dry cell weight when grown on glucose. Previous studies indicated that other C. echinulata strains could transform complex aromatic compounds. ${ }^{19}$ However, despite these promising findings, this particular strain has not been used to convert lignocellulosic biomass, and neither has oleaginous fungus been evaluated for its lignin degradation capacity during biomass conversion. Third and the most importantly, considering the potential to utilize lignin, this unique oleaginous strain may be used to convert biomass directly without chemiphysical pretreatment. Even though different conversion platforms were developed to process different components of the plant cell wall, few platforms can actually convert lignin together with carbohydrate into fungible fuels and products via biological processes. This study aims to offer an alternative approach where a simple biological system could convert all the components of the plant cell wall into biofuel products without additional pretreatment processes.

Therefore, we evaluated the possibility of such a system with three aspects of studies. First, we evaluated whether the oleaginous fungus C. echinulata FR3 can convert lignin while processing carbohydrate. Second, we investigated whether lignin modification of feedstock can be integrated with this oleaginous fungus strain to improve the efficiency of biomass conversion without chemical pretreatment. Third, we further investigated the mechanisms for the lignin utilization by oleaginous fungi using transcriptomics and chemical analysis. The discovery indicated that oleaginous fungi like FR3 can be exploited for complete biomass utilization in a consolidated platform without chemical pretreatment or can be enhanced to convert lignin waste into lipid. These novel oleaginous fungus-based platforms could have profound impacts on energy and environmental sustainability when implemented.

\section{Materials and supplies}

\subsection{Microorganism maintenance and spore inoculum preparation}

C. echinulata FR3 was isolated by Key Laboratory of Molecular Biophysics at Huazhong University of Science and Technology and identified by China General Microbiological Culture Collection Center in Beijing. The strain was maintained at $4{ }^{\circ} \mathrm{C}$ on potato dextrose agar (PDA) slants. To prepare the spore inoculum, the fungal mycelium was incubated on PDA in $250 \mathrm{~mL}$ Erlenmeyer flasks at $28{ }^{\circ} \mathrm{C}$ for 5 days to form spores. The spores were then collected by washing the mycelium with $10 \mathrm{~mL}$ sterile water and transferred to $100 \mathrm{~mL}$ of potato dextrose broth (PDB) medium in $250 \mathrm{~mL}$ Erlenmeyer flasks with a final spore concentration of $3 \times 10^{7}$ spores $\mathrm{L}^{-1}$. The inoculation was cultivated at $28{ }^{\circ} \mathrm{C}$ with a shaking speed of $150 \mathrm{rpm}$ for $36 \mathrm{~h}$.

\subsection{Sorghum biomass}

The single- and double-mutant sorghum stocks were developed as previously described. ${ }^{21}$ The wild-type and mutant lines of sorghum were grown in randomized block design at University of Nebraska Agricultural Research and Development fields near Mead, NE in 2010. The samples consisted of bulk biomass harvested from 4 plots. The grain heads were removed and sorghum lignocellulosic tissue (leaves and stalks) was dried at $50{ }^{\circ} \mathrm{C}$ in an air-blowing oven for 3 days. The tissue was ground using a Wiley® mill (2 mm screen; Arthur H. Thomas Co., Philadelphia, PA).

\subsection{Dilute-acid pretreatment}

The dilute-acid pretreatment of sorghum was carried out by mixing with diluted sulfuric acid to a final concentration of $1.5 \%(\mathrm{w} / \mathrm{w})$ at a solid loading of $1: 17(\mathrm{w} / \mathrm{v})$. The mixture was maintained at $121{ }^{\circ} \mathrm{C}$ for $1 \mathrm{~h}$. After the reaction, 
the samples were neutralized to $\mathrm{pH} 7.0$ with $2 \mathrm{M}$ sodium hydroxide.

\subsection{Enzymatic hydrolysis and fungal conversion}

For untreated sorghum stover enzymatic hydrolysis, samples were adjusted with distilled water to a solid loading of $1: 17$ $(\mathrm{w} / \mathrm{v})$ and autoclaved at $121{ }^{\circ} \mathrm{C}$ for $20 \mathrm{~min}$ with a liquid cycle. Different cellulase enzyme (Sigma, C2730) loadings (3.0, 4.5 and 6.0 FPU per g sorghum) were used for hydrolysis at $28{ }^{\circ} \mathrm{C}$ on a rotary shaker incubator at $150 \mathrm{rpm}$ for $48 \mathrm{~h}$. For the acidpretreated wild-type sorghum, the cellulase was directly added to the neutralized sample with an enzyme loading of $4.5 \mathrm{FPU}$ per $\mathrm{g}$ sorghum biomass. The hydrolysis reactions were conducted as aforementioned. After hydrolysis, the pre-cultured C. echinulata FR3 were inoculated at a $5 \%(\mathrm{v} / \mathrm{v})$ fungal biomass loading, and cultured on a rotary shaker incubator at $28{ }^{\circ} \mathrm{C}$ and $150 \mathrm{rpm}$.

\subsection{Sorghum biomass and mycelium biomass evaluation}

The sorghum biomass and the mycelium biomass from the fermentation were harvested every 72 hour period $(0,3,6$ and 9 days) by centrifugation. The fungal mycelium pellets were separated from the sorghum biomass manually, and both the sorghum and fungal mycelium were washed with distilled water twice and dried at $60{ }^{\circ} \mathrm{C}$ for three days to a constant weight. After drying, the weight of both the sorghum and fungal mycelium was measured and recorded.

Lignin (cellulose, hemicellulose) loss (\%) =

100 - Lignin (cellulose, hemicellulose) content in converted sorghum $\times$ Final weight of sorghum after conversion Lignin (cellulose, hemicellulose) content in raw sorghum $\times$ Initial weight of raw sorghum

\subsection{Measurement of reducing sugar, cellulase and xylanase activity}

The cultivation supernatant was collected by centrifugation to measure the reducing sugar concentration, cellulase, and xylanase activities. Reducing sugar was measured with the DNS (3,5-dinitrosalicylic acid) reagent using the absorbance at $540 \mathrm{~nm}$ with a glucose standard curve. ${ }^{22}$ The cellulase activity in the medium was assayed based on the 'filter-paper-unit (FPU)' following the Laboratory Analytical Procedure (LAP 006) from the National Renewable Energy Laboratory (NREL). The xylanase activity was assayed using xylan as the substrate to measure the release of reducing sugar by the DNS method by a reaction for $30 \mathrm{~min}$ at $37^{\circ} \mathrm{C}$ and $\mathrm{pH} 4.8$. One unit of xylanase activity was calculated as $1 \mu \mathrm{mol}$ of reducing sugar from xylan per min under the reaction conditions.

\subsection{Lipid production evaluation}

The extraction and composition analysis of total lipid were achieved by GC/MS (gas chromatography/mass spectrometry) according to the protocol described in our previous study. ${ }^{23}$ The total lipid of the fungal mycelium was extracted in the form of fatty acid methyl ester (FAME) by the sulfuric acidmethanol method and collected in pre-weighed tubes. The tube with the extracted FAME was further dried to a constant weight and then weighed to derive the lipid yield.

The lipid composition was analyzed by GC/MS using an Agilent 7890 GC (Agilent Technologies, Santa Clara, CA) coupled with an Agilent 5975 mass spectrometer. An HP-5MSI column was used. The injection port was kept at $280{ }^{\circ} \mathrm{C}$ and the MS transfer line was set to $100{ }^{\circ} \mathrm{C}$. The GC oven temperature was programmed as follows: initially at $40{ }^{\circ} \mathrm{C}(0.5 \mathrm{~min})$, increased to $110{ }^{\circ} \mathrm{C}$ at $5{ }^{\circ} \mathrm{C} \mathrm{min}^{-1}$, and then increased from 110 to $300{ }^{\circ} \mathrm{C}$ at $20{ }^{\circ} \mathrm{C} \mathrm{min}^{-1}$ for a total run time of $24 \mathrm{~min}$. The raw chromatography and mass spectral data were processed with the software Enhanced ChemStation (Agilent Technologies, Santa Clara, CA), and the quantity of the specific lipid molecules was analyzed by the peak area.

\subsection{Compositional analysis}

The structural carbohydrate (cellulose and hemicellulose), lignin, and ash content of sorghum stovers was determined according to the Laboratory Analytical Procedure from the National Renewable Energy Laboratory (NREL). ${ }^{24}$ Carbohydrate concentrations were analyzed using high-performance liquid chromatography (HPLC 1260 Infinity; Agilent Technologies, CA). HPLC analyses were carried out with an Aminex HPX-87P column (300 mm by $7.8 \mathrm{~mm}$; Bio-Rad Laboratories, CA) with HPLC grade water as the mobile phase and a flow rate of $0.6 \mathrm{~mL} \mathrm{~min}^{-1}$ at $85{ }^{\circ} \mathrm{C}$ using an RI detector. The lignin, cellulose and hemicellulose degradation efficiencies were calculated as follows:

\subsection{Solid state ${ }^{13} \mathrm{C} \mathrm{CP} / \mathrm{MAS} \mathrm{NMR}$ analysis}

The carbon-13 cross-polarization (CP) magic angle spinning (MAS) $\left({ }^{13} \mathrm{C} \mathrm{CP} / \mathrm{MAS}\right)$ solid-state $\mathrm{NMR}$ was carried out to confirm the compositional and chemical changes of the structural components for sorghum biomass under different treatments. The control biomass tissue $(250 \mathrm{mg}$ ) and samples after conversion were individually packed in a $5 \mathrm{~mm}$ pencil-type rotor and the spectra were recorded under identical acquisition parameters. The solid state ${ }^{13} \mathrm{C} \mathrm{CP} / \mathrm{MAS}$ analyses were carried out at $100 \mathrm{MHz}$ on a Bruker Avance 400 spectrometer (NMR Center, Washington State University), equipped with a Chemagnetics double resonance probe. A contact time of $0.5 \mathrm{~ms}$, a proton field of $c a .40 \mathrm{kHz}$ during $\mathrm{CP}$ and data acquisition, a relaxation delay of $4 \mathrm{~s}$ and a spinning speed of $5 \mathrm{kHz}$ were applied to obtain the ${ }^{13} \mathrm{C} \mathrm{CP} / \mathrm{MAS}$ spectra. All the corresponding ${ }^{13} \mathrm{C} \mathrm{CP} / \mathrm{MAS}$ spectra were derived from 17500 scans, with the chemical shifts given in $\delta$ ppm. The integrals for each resonance and/or chemical shift value arising from the cell wall components of fungal converted biomass were normalized with reference to their corresponding control spectra for semi-quantitative analysis. 


\subsection{Transcriptomics analysis}

The total RNA was extracted from C. echinulata FR3 grown on sorghum for 6 days with a Qiagen RNeasy Plant Mini Kit. The transcriptomics sequencing of the fungal samples was performed using Illumina HiSeq 2500 by the Institute of Plant Genomics and Biotechnology, Texas A\&M University. The resulting reads were assembled with Trinity. ${ }^{25}$ To further reduce the redundancy of the assembled transcripts, the sequence clustering package CD-HIT was used to cluster similar sequences that meet similarity threshold $>0.9$ into UniGene clusters. ${ }^{26}$ UniGenes were then functionally annotated based on the UniProtKB (http://www.uniprot.org/) database by the package of ncbi-blast-2.2.28+ with the threshold of $e<10^{-6}$. To quantify the expression level of UniGenes, sequencing reads were mapped to the UniGene sequences. Gene expression abundance was calculated as normalized mapping read counts per million total mapping reads per $\mathrm{kb}$ of the gene length. The assembled UniGenes were also aligned to the CAZy database based on sequence similarity to carry out the lignocellulolytic degradation functional analysis through the Bioenergy Science Center web service (http://mothra. ornl.gov/cgi-bin/cat/cat_v2.cgi?tab=ORTHOLOGS) with $E$-value threads $\leq 10^{-6}$ and Pfam domain bit score threshold $\geq 100$. The phylogenetic analysis was based on PhyMl through the Phylogeny.fr web server. ${ }^{27,28}$

\subsection{Determination of iron-reducing activity}

The iron-reducing activity was determined based on the formation of a ferrozine- $\mathrm{Fe}^{2+}$ complex. $^{22} 2 \mathrm{~mL}$ of cultivation supernatants collected by centrifugation were mixed with $0.5 \mathrm{~mL} \mathrm{FeCl}_{3}(1.2 \mathrm{mM})$ and $0.5 \mathrm{~mL}$ ferrozine $(15 \mathrm{mM})$, and the absorbance change at $562 \mathrm{~nm}$ was immediately measured using a UV-visible spectrometer for $3 \mathrm{~min}$. 1 unit $(A / \mathrm{min})$ of iron-reducing activity was defined as the rate of absorbance increase at $562 \mathrm{~nm}$ per minute.

\section{Results and discussion}

3.1. C. echinulata FR3 (FR3) can degrade all cell wall components including cellulose, hemicellulose, and lignin

Biomass composition analysis confirmed the reduced lignin content in the bmr lines (Table $\mathrm{S} 1 \dagger$ ). ${ }^{13,29}$ C. echinulata FR3 fermentation led to the degradation of all cell wall structural components including lignin. Relatively lower cellulase loadings (3.0, 4.5 and 6.0 FPU per g sorghum) were used for saccharification (Fig. S1†), because oleaginous fungi can secrete cellulases for saccharification. ${ }^{16,23}$ Both the bmr6 and the bmr6/bmr12 double mutants allowed higher saccharification efficiency than that of wild-type (Fig. S1†). The sorghum biomass hydrolyzed with 4.5 FPU per $\mathrm{g}$ enzyme loading was then subjected to $C$. echinulata FR3 fermentation to evaluate the degradation of different cell wall components. As shown in Fig. 1A, weight loss was observed for cellulose, hemicellulose and lignin during the first six days of fermentation. The weight loss reached a plateau at Day 6 to Day 9 for most of the samples. In general, a higher percentage of weight loss was achieved for all mutants as compared to wild-type (Fig. 1).
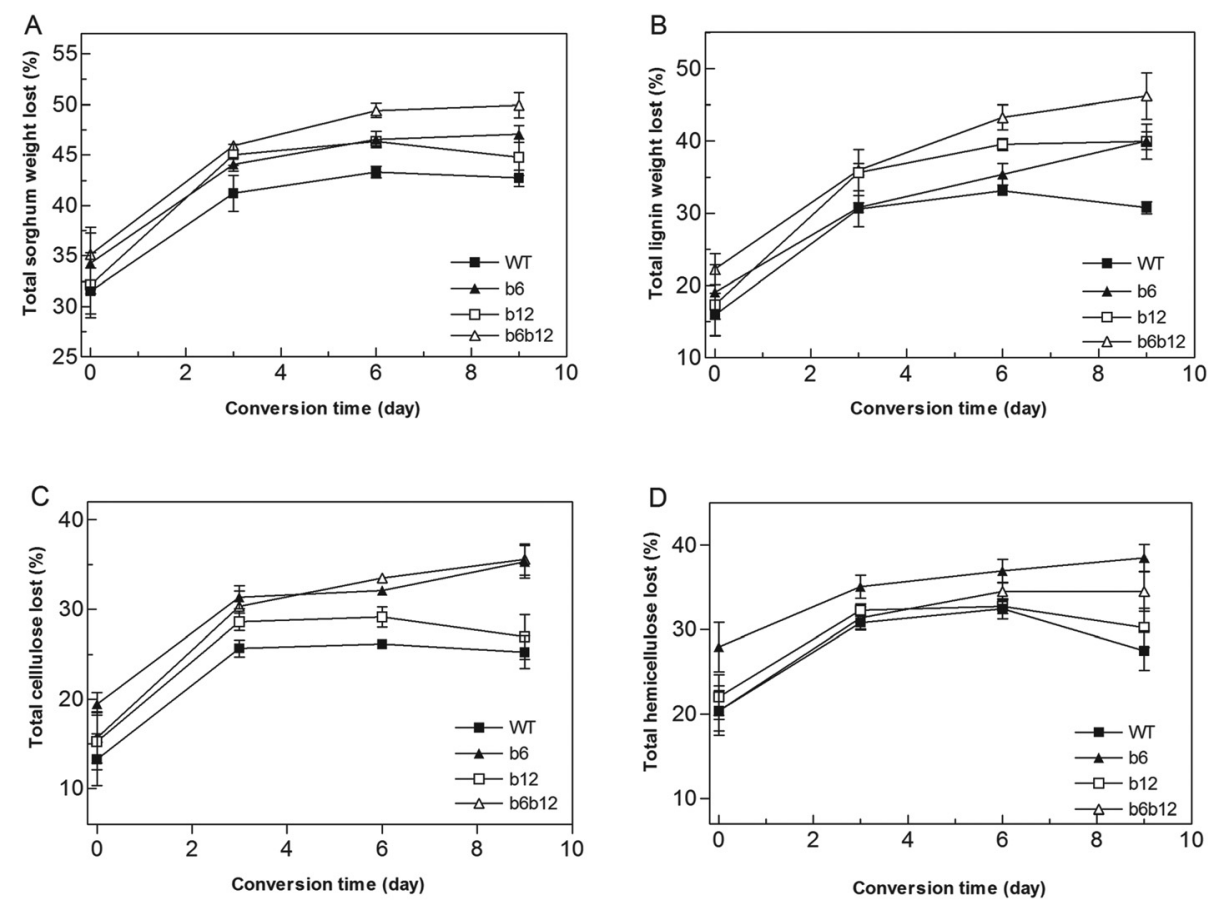

Fig. 1 Percentage of weight loss for total sorghum straw (A), lignin (B), cellulose (C) and hemicellulose (D) during the conversion of wild-type (solid square), bmr6 mutant (solid triangle), bmr12 mutant (open square) and bmr6/bmr12 double mutant (open triangle) sorghum biomass by $C$. echinulata FR3. The percentages of weight loss as compared to the original composition in raw sorghum biomass were recorded to represent the composition degradation efficiencies. 

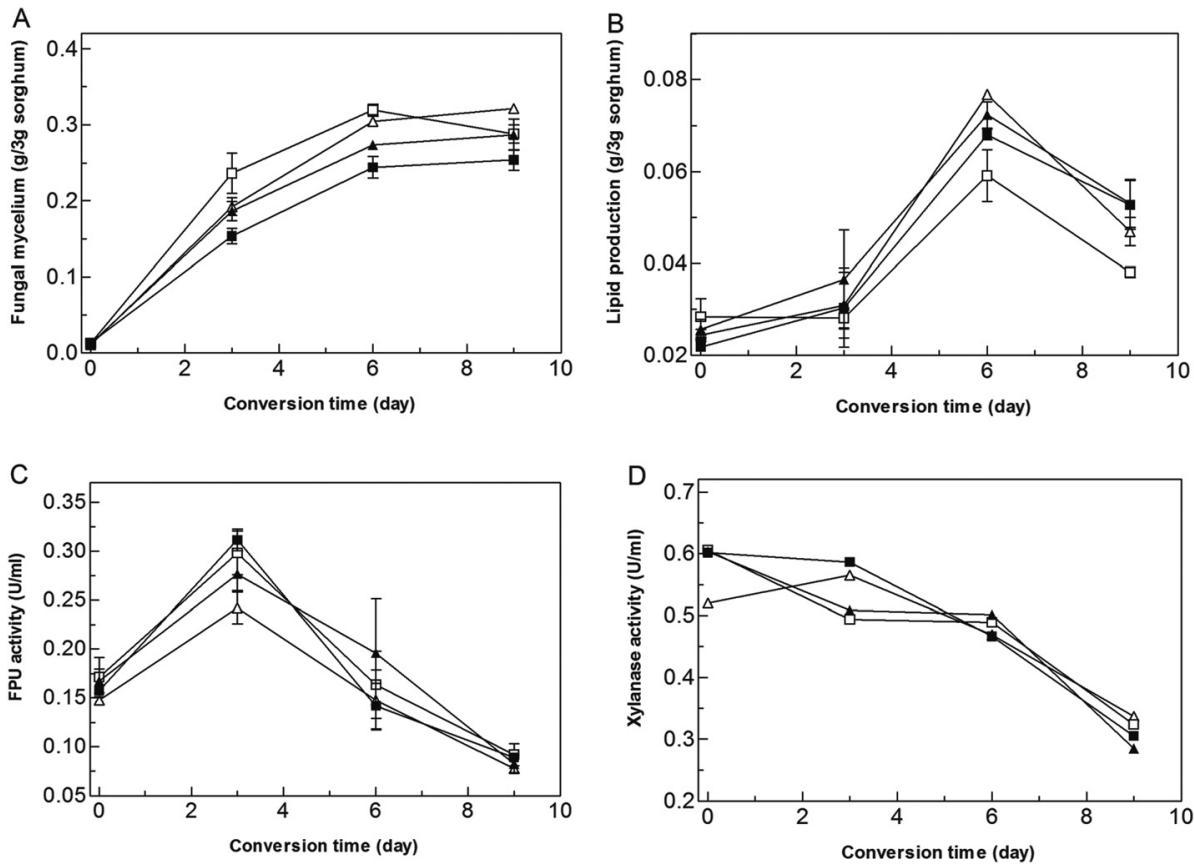

Fig. 2 Mycelium growth (A) and lipid accumulation (B) of C. echinulata FR3 grown in sorghum biomass. FPU activity (C) and xylanase activity (D) of the cultivation supernatant variations during the conversion of wild-type (solid square), bmr6 mutant (solid triangle), bmr12 mutant (open square) and bmr6/bmr12 double mutant (open triangle) sorghum biomass by $C$. echinulata FR3. All assays were carried out in triplicates.

In particular, more significant lignin weight loss was found for both bmr12 and bmr6/bmr12 double mutants. For example, the lignin weight loss for double mutant and wild-type was $46 \%$ and $31 \%$, respectively (Fig. 1B). More significant cellulose weight loss was observed for both bmr6 and the bmr6/bmr12 double mutants (Fig. 1C). The fermentation of bmr6 mutant led to the most significant hemicellulose weight loss (Fig. 1D). The significant degradation of cell wall lignin in C. echinulata FR3 is noteworthy as the phenomenon is normally common to Basidiomycetes, but not to other genera of fungi. As an oleaginous fungus, the capacity to degrade lignin over $30 \%$ offers C. echinulata FR3 new opportunities of the utilization of multiple cell wall components for useful products and the bioconversion without chemical pretreatment.

\subsection{Lignin modification promoted biomass conversion by C. echinulata FR3}

The biomass weight loss correlated well with the growth of C. echinulata FR3. In general, C. echinulata FR3 grew faster and accumulated more fungal biomass on $b m r$ mutants, which was consistent with the greater sorghum biomass weight loss in bmr mutants (Fig. 2A). The fungal cell growth reached plateau phase at Day 6, when the sorghum biomass weight loss also turned to plateau phase. Among the three mutant lines of sorghum, FR3 grown on bmr6/bmr12 double mutant and bmr12 mutant accumulated higher cell biomass as compared to that of bmr6 and wild-type (Fig. 2A).

The weight loss and fungal growth also correlated with the lipid yield. The lipid yield for all fermentation experiments increased during the first six days and decreased on Day 9
(Fig. 2B). The result could be explained by the fact that both fungal growth and sorghum biomass weight loss reached a plateau at Day 6. The fungus might start catabolizing lipids after Day 6, because the reduced sugar also reached plateau phase at Day 6 (Fig. S2 $\dagger$ ). Day 6 thus represented the optimal time to harvest and compare lipid yields among different lines. Among the three bmr lines, both bmr6 and bmr6/bmr12 double mutants yielded more lipid during fermentation. The fermentation of bmr6/bmr12 led to the most significant increase in lipid production.

In order to further elucidate the mechanisms for biomass degradation, we analyzed reduced sugar and enzyme activities in the fermentation system. As aforementioned, the level of reduced sugar dropped rapidly during the first six days of fermentation and reached a plateau at Day 6 to Day 9 (Fig. S2 $\dagger$ ). The enzyme activity reached a peak level at Day 3 (Fig. 2C and D). Considering that the exogenous cellulase was added two days before Day 0, the result clearly indicated that endogenous cellulase played an important role in the biomass degradation and fungal cell growth. The results of enzyme and reducing sugar analysis were consistent with the fungal growth and lipid yield. Besides the cellulose and hemicellulose, a detailed analysis of lignin content was carried out using NMR spectroscopy to further confirm lignin degradation.

\subsection{NMR analysis confirmed the degradation of lignin along with carbohydrates}

In order to further confirm that $C$. echinulata FR3 could convert lignin along with the carbohydrates, the solid-state NMR was carried out to analyze sorghum biomass using the 
Table 1 Semi-quantitative analysis of cell wall components by ${ }^{13} \mathrm{C} \mathrm{CP/MAS} \mathrm{NMR} \mathrm{analysis.} \mathrm{WT-raw:} \mathrm{wild-type} \mathrm{sorghum} \mathrm{without} \mathrm{fungal} \mathrm{conversion;}$ WT- 6 days: wild-type sorghum after 6 days fungal conversion; b6b12-raw: bmr6/bmr12 double mutant sorghum without fungal conversion; b6b126 days: bmr6/bmr12 double mutant sorghum after 6 days fungal conversion

\begin{tabular}{|c|c|c|c|c|c|c|}
\hline WT-raw & 66.7 & 16.8 & 3.3 & 3.7 & 6.5 & 4 \\
\hline b6b12-raw & 63.4 & 18.6 & 3.2 & 5.3 & 6.2 & 3.4 \\
\hline b6b12-6 days & 73.4 & 13.0 & 2.4 & 3.7 & 3.9 & 5.7 \\
\hline
\end{tabular}

conventional CP/MAS (cross-polarization-magic angle spinning) method. ${ }^{30}$ The analysis was focused on the structural changes of the wild-type and bmr6/bmr12 double mutant sorghum after 6 days fungal conversion as compared to the reference samples without fermentation. The normalized ${ }^{13} \mathrm{C}$ $\mathrm{CP} /$ MAS NMR spectra are depicted in Fig. S3 and Fig. S4† and the most predominant assignments for each resonance peak are listed in Table $S 2 . \dagger^{31,32}$ The acquired spectra mainly contained chemical resonance values analogous to the carbohydrate region (60-100 ppm) and the aromatic region (100-162 ppm). In addition, the chemical shift resonances of carbonyl and carboxyl groups (160-200 ppm), the methoxyl group (52-55 ppm) and carbon in the etherified and/or nonetherified region (132-152 ppm) were also observed. The comparison of ${ }^{13} \mathrm{C} \mathrm{CP} / \mathrm{MAS}$ NMR spectra among different samples revealed several features.

First, comparison of aromatic carboxyl resonances (165-175 ppm) indicated the significant differences in the lignin derived aromatic region (Fig. S4A $\dagger$ ). The results suggested that FR3 conversion of sorghum biomass resulted in lignin utilization, confirming the lignin weight loss data. Second, the ${ }^{13} \mathrm{C}$ CP/MAS NMR analysis showed reduced intensity in the aliphatic carbonyl/carboxyl group resonances (175-185 ppm) for both wild-type and bmr6/bmr12 mutant after conversion by $C$. echinulata FR3. The result strongly indicated the removal of hemicelluloses and side chain alterations in the lignin macromolecular assembly, ${ }^{33}$ which could result from cell wall deconstruction by $C$. echinulata FR3 during the 6 days conversion process. Third, in contrast to the aforementioned decreases, a slight increase in the intensity at chemical shift values of 60-90 ppm (carbohydrate region) was observed in the biomass samples after fungal conversion. Such an increase was due to the relatively more rapid reduction in the content of aromatic lignin and aliphatic carbon. The result suggested that the lignin degradation was more rapid than cellulose degradation during the six days of conversion by C. echinulata FR3. The result was consistent with the composition analysis. The quantification of resonances with reference to the control ${ }^{13} \mathrm{C} \mathrm{CP} / \mathrm{MAS}$ NMR spectra was also carried out under normalized conditions to determine the content (in \%) of cell wall components in the corresponding samples (Table 1). The results further confirmed the degradation of all cell wall components including cellulose, hemicellulose and lignin by $C$. echinulata FR3 conversion. In addition, Table 1 further confirmed more rapid degradation of lignin, as the aro- matic contents in wild-type and bmr6/bmr12 mutant after conversion were reduced to $12.1 \%$ and $13.0 \%$, respectively, as compared to $16.8 \%$ and $18.6 \%$ in the untreated controls. Overall, the biomass composition analysis and ${ }^{13} \mathrm{C} C P / M A S$ NMR analysis were highly consistent to demonstrate that C. echinulata FR3 could degrade all the plant cell wall components including lignin, cellulose, and hemicellulose.

\subsection{A similar lipid yield can be achieved for the fermentation of the $b m r$ mutant and acid-pretreated wild-type biomass}

The consumption of lignin by the oleaginous fungus suggested that $C$. echinulata FR3 conversion of sorghum biomass might alter the lignin content and structure, in a process similar to biological pretreatment by the white rot fungus. We therefore hypothesized that biomass conversion might be carried out without chemical pretreatment, if biomass has a relatively low recalcitrance. We further compared the fungal growth and lipid yield of $C$. echinulata FR3 grown on acid-pretreated wildtype and non-pretreated bmr6/bmr12 double mutant sorghum stover after 6 days (Fig. 3A). Even though acid-pretreated wildtype sorghum led to a much higher weight loss $(65.5 \%)$ than non-pretreated bmr6/bmr12 sorghum (Fig. 3B), no significant differences in the cell growth and lipid production were found between the two types of samples (Fig. 3A). The greater weight loss might be caused by the degradation of hemicellulose by sulfuric acid during the pretreatment (Fig. 3C). The GC/MS analysis also revealed that the lipid profile of $C$. echinulata FR3 has no significant difference between the two conditions (Fig. S5 $\dagger$ ). It is well established that various inhibitors and toxins could be generated during the dilute acid pretreatment to inhibit microbial growth. ${ }^{34,35}$ The inhibitors and toxins might also inhibit the cell growth and cause low lipid yield during biomass conversion by the oleaginous fungus C. echinulata FR3.

\subsection{Transcriptomics analysis revealed the lignin and biomass degradation mechanism in FR3}

Even though some filamentous fungi could degrade the lignocellulosic biomass, few studies indicated that any non-basidiomycetous filamentous fungi could degrade lignin. In particular, the lignin degradation capacity in oleaginous fungi might be exploited for broad applications including complete biomass utilization, consolidated biomass processing, and lignin conversion to lipid. The development of these new 

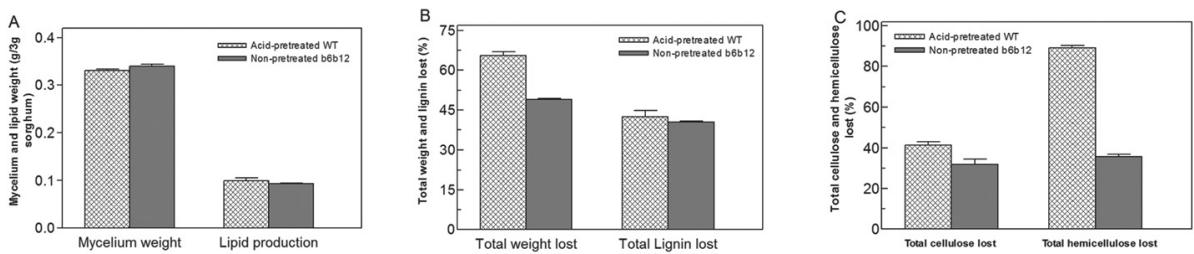

Fig. 3 (A) Comparison of mycelium growth and lipid accumulation of $C$. echinulata FR3 grown on acid-pretreated wild-type (WT) sorghum straw and non-pretreated bmr6/bmr12 double mutant (b6b12) sorghum straw for 6 days. (B) Comparison of total sorghum weight and lignin loss during the conversion of acid-pretreated wild-type and non-pretreated double mutant sorghum straw by C. echinulata FR3 after 6 days. (C) Comparison of cellulose and hemicellulose degradation during the conversion of acid-pretreated wild-type and non-pretreated double mutant sorghum straws by C. echinulata FR3 after 6 days.

platforms will depend on an in-depth understanding of biomass and lignin degradation mechanisms in C. echinulata FR3. A transcriptomics analysis of FR3 grown on the wild-type and the bmr6/ bmr12 mutant sorghum straw was carried out to further explore the mechanisms for biomass, in particular, lignin degradation.

The result revealed that $C$. echinulata FR3 synergized extracellular lignocellulolytic enzymes and radical systems for biomass degradation. The transcriptomics analysis revealed three features. First, FR3 had cellulase enzymes, but not in a balanced combination to achieve maximized cellulose and hemicellulose degradation (Fig. 4A). The analysis based on the CAZy database revealed that cellulose and hemicellulose degradation enzymes were expressed, which was consistent with the cellulase and xylanase activities detected. Among the 17312 predicted gene models, 502 belonged to different CAZy families (bit score $\geq 100$ ), including 131 glycoside hydrolases (GH) and 55 carbohydrate esterases (CE) (Fig. S6†). Despite the prevalence of GH enzymes, only 2 endoglucanases, 2 beta-1,4glucosidases and 1 exoglucanase were identified. Among these cellulolytic enzymes, only CeFR966 encoding endoglucanase (GH9) was expressed at a high level (Fig. 4A). The result indicated that $C$. echinulata FR3 had a carbohydrate degradation capacity, yet the capacity is limited as compared to most of the other cellulose degradation fungi.

Second, an alternative extracellular lignolytic enzyme system was identified in C. echinulata FR3 (Fig. 4B). The enzyme system is slightly different from the classic lignolytic enzyme system based on peroxidases and laccases in white rot fungi. ${ }^{36}$ Several gene models were annotated to be multiple copper oxidases, and one of them, CeFR1943, encoded a laccase-like multiple copper oxidase (LMCO) with a secretion signal peptide sequence. A phylogenetic analysis of this LMCO with classic laccases from fungi (ascomycetes and basidiomycetes) and plants revealed that the enzyme was related to basidiomycete fungal laccases known for a strong lignin degradation capacity. The results indicated that the LMCO might be involved in lignin depolymerization in C. echinulata FR3. Meanwhile, another gene CeFR715 encoded aromatic peroxygenase (APO) with predicted secretive signal peptides. Extracellular APOs are a novel group of heme-thiolate enzymes characterized for lignin degradation by catalyzing $\mathrm{H}_{2} \mathrm{O}_{2}$-dependent oxidative cleavage of diverse ethers in aromatic substrates. ${ }^{37-39}$ The transcriptomics analysis thus suggested that LMCO and APO might be the main extracellular lignolytic enzymes for $C$. echinulata FR3.

Third, a strong radical generation system was identified for C. echinulata FR3 and the radicals can synergize either with the aforementioned oxidative enzymes or with the ironquinone system for the Fenton reaction. Many enzymes contributing to extracellular $\mathrm{H}_{2} \mathrm{O}_{2}$ generation were discovered from the transcriptomics analysis (Fig. 4B). Copper radical oxidase (CRO) and GMC oxidoreductase were two major groups involved in extracellular $\mathrm{H}_{2} \mathrm{O}_{2}$ generation for the Fenton system in the most studied fungi system. ${ }^{40-42}$ Unlike the basidiomycetes expressing several CROs, only one CRO, CeFR251, was identified in $C$. echinulata FR3 encoding galactose oxidase with predicted secretive signal peptides. Moreover, this gene was expressed in a very high level as compared to many other genes (Fig. 4B). In the meanwhile, several GMC oxidoreductase-type genes were identified encoding long-chain-alcohol oxidase. These results indicated that $C$. echinulata FR3 thus had a system to generate extracellular $\mathrm{H}_{2} \mathrm{O}_{2}$.

Fourth, the identification of iron reduction related proteins further suggested that the radical generation synergized with the Fenton reaction (Fig. 4B). Besides the extracellular enzymes, wood degradation by basidiomycetes often involved extracellular low molecular weight oxidants, particularly Fenton reaction generated hydroxyl radicals. ${ }^{40,43-45}$ The transcriptomics analysis suggested that $C$. echinulata FR3 also had a similar Fenton reaction-based radical system for lignin depolymerization. Several gene models (CeFR296, CeFR2458, CeFR 2745 and CeFR8451) were identified as putative quinone reductases (QRD) and were expressed in a relatively high level. Redox cycling of secreted quinones was considered as a driving force for the Fenton reaction in wood-degrading fungi. ${ }^{46,47}$ The expression of these putative QRD in coordination with quinate permeases (CeFR7596 and CeFR10458) for the biosynthesis and transport of quinones might enable an efficient extracellular Fenton system. In addition to the quinone-based iron reduction system, transmembrane ferric reductase (CeFR10940) was also found expressed with a possible $\mathrm{Fe}^{3+}$ reduction function.

Overall, the transcriptomics analysis revealed a unique lignocellulose degradation system in C. echinulata FR3 

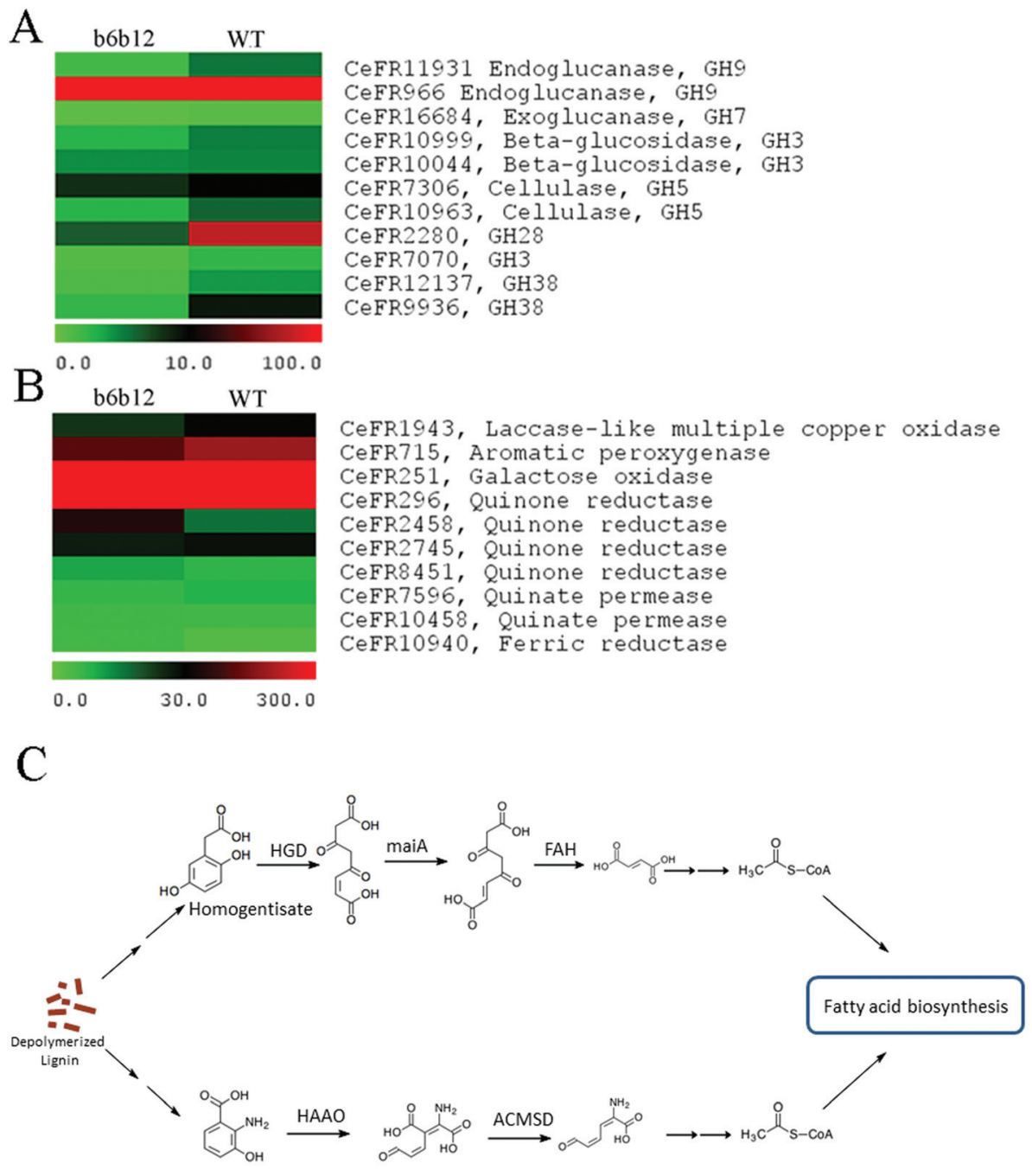

3-Hydroxyanthranilate

Fig. 4 Transcriptomics analysis to reveal the lignocellulose degradation mechanisms in C. echinulata FR3. (A) Expression abundance of selected cellulases and hemicellulases. (B) Expression abundance of selected extracellular lignolytic enzymes and Fenton system related enzymes. (C) Central aromatic compound catabolism pathway in C. echinulata FR3. HGD, homogentisate 1,2-dioxygenase; maiA, maleylacetoacetate isomerase; FAH, fumarylacetoacetase; HAAO, 3-hydroxyanthranilate 3,4-dioxygenase; ACMSD, aminocarboxymuconate-semialdehyde decarboxylase.

by synergizing extracellular lignocellulolytic enzymes and hydroxyl radicals (Fig. 5). Such a system has enabled C. echinulata FR3 to become one of the few known strains outside of basidiomycetes with an appreciable lignin degradation capacity. During biomass degradation, C. echinulata FR3 could express extracellular $\mathrm{H}_{2} \mathrm{O}_{2}$-generation related oxidoreductases (mainly galactose oxidase) and ferric reduction related proteins (QRD and ferric reductase) to mediate the Fenton reaction $\left(\mathrm{Fe}^{2+}+\mathrm{H}_{2} \mathrm{O}_{2} \rightarrow \mathrm{Fe}^{3+}+{ }^{\circ} \mathrm{OH}+\mathrm{OH}^{-}\right)$. The generated hydroxyl radicals from the Fenton-based system could both depolymerize polysaccharides and lignin to make the lignocellulose more accessible for lignocellulolytic enzymes. ${ }^{40,44,48}$ For lignin depolymerization, extracellular APO and LMCO could synergize with hydroxyl radicals to carry out efficient lignin depolymerization in a way different from basidiomycetes. For cellulose and hemicellulose degradation, the enzymatic cellulose degradation pattern of C. echinulata FR3 was very similar to many of the brown rot fungi, in which endoglucanases were highly expressed whilst cellobiohydrolases were poorly expressed or even absent during the cellulose degradation. ${ }^{49}$ After the lignin depolymerization process, the released small molecular aromatic compounds could be transported into the cell for complete aromatic compound catabolism. Pathway analysis revealed two central aromatic catabolism pathways involving the ring-cleavage of homogentisate and 3-hydroxyanthranilate (Fig. 4C). ${ }^{50}$ Numerous cytochrome P450s $(\sim 50$ genes $)$ were also discovered in C. echinulata FR3, indicating that cytochrome $\mathrm{P} 450$ s may be involved in degradation/conversion of the lignin derived metabolites into central aromatic intermediates for complete degradation as previously reported..$^{51,52}$ 


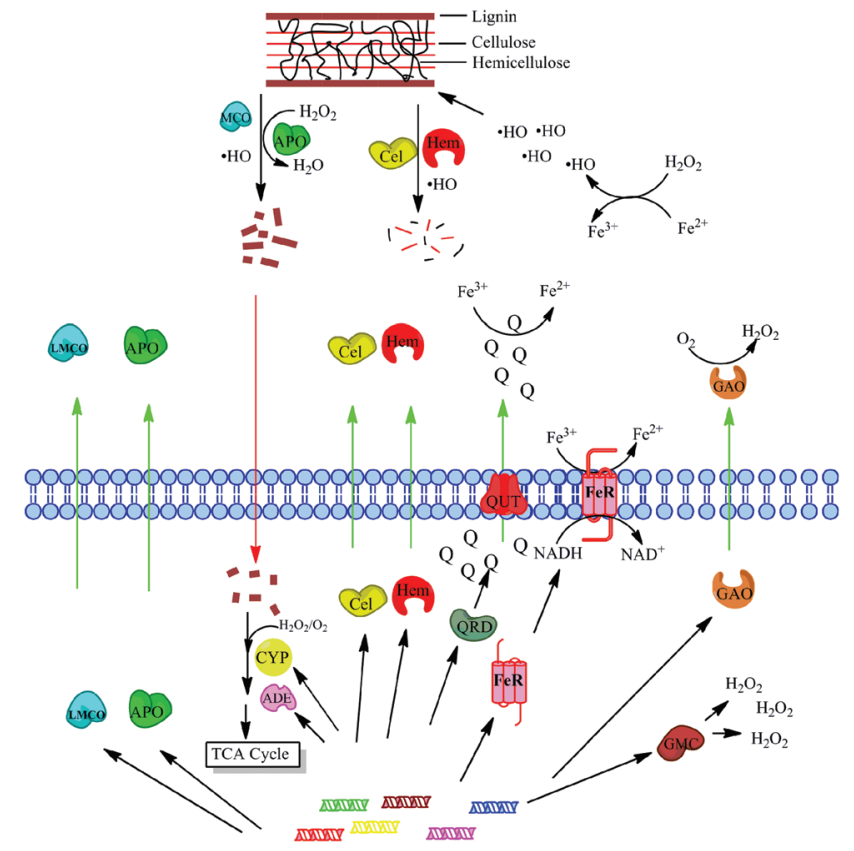

Fig. 5 An integrated model to elucidate the lignocellulose degradation mechanisms in C. echinulata FR3. LMCO, laccase-like multiple copper oxidase; APO, aromatic peroxygenase; CYP, cytochrome P450; Cel, cellulase; Hem, hemicellulase; $Q R D$, quinone reductase; FeR, ferric reductase; GAO, galactose oxidase; GMC, GMC oxidoreductase; QUT, quinate permeases; $A D E$, aromatic compound degradation enzymes.

\subsection{Enzyme assay further confirmed that the Fenton reaction is one of the mechanisms for lignin degradation in C. echinulata FR3}

In order to further verify the role of radicals and Fenton reaction in lignin degradation, iron-reducing activity was measured as an indicator for hydroxyl radical levels. The hydroxyl radical level is often impacted by iron reducing capacity, which is important in Fenton reaction. As shown in Fig. 6, the iron-reducing activity was significantly increased in the C. echinulata FR3 grown on biomass as compared to that on PDB medium. No significant difference was observed in iron

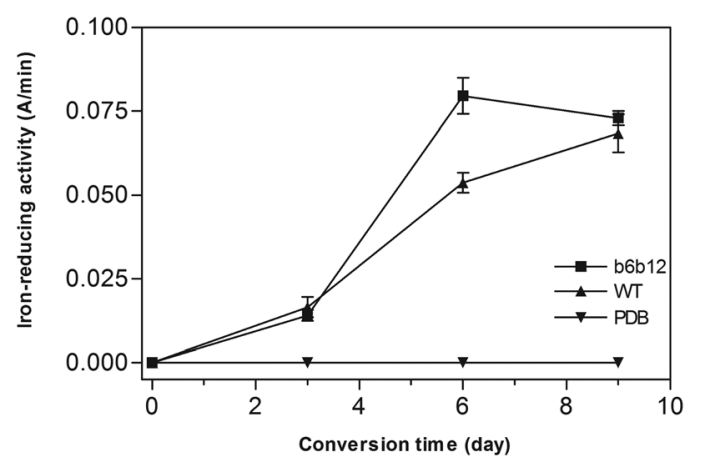

Fig. 6 Measurement of the iron-reducing activity of the cultivation supernatant of $C$. echinulata FR3 grown on wild-type (solid triangle) sorghum biomass, bmr6/bmr12 double mutant (solid square) sorghum biomass, and PDB (solid inverted triangle). reducing activity of fungi grown on wild-type and bmr6/bmr12 mutant sorghum. The significant induction of iron-reducing activity throughout the biomass fermentation process indicated that the induced Fenton reaction system might play an important role in lignin and biomass degradation, confirming the transcriptomics data.

\subsection{Perspectives on oleaginous fungi with a strong lignin degradation capacity}

Overall, the biomass composition, chemical, and transcriptomics data all suggested that the oleaginous fungus C. echinulata FR3 has a unique capacity to efficiently utilize lignin. The discovery of an oleaginous fungus with a strong lignin degradation capacity could be important from several perspectives. First, even though lignin degradation by fungi has been studied extensively in basidiomycetes, few studies indicated that the capacity exists in filamentous fungi beyond basidiomycetes. From a scientific perspective, the genomic and molecular mechanisms for lignin degradation in C. echinulata FR3 and its relatedness to the mechanisms in basidiomycetes indicated that additional species evolved a lignin degradation capacity in a convergent way using different mechanisms. From the application perspective, the oleaginous fungus with a lignin degradation capacity will bridge an important limitation as the white rot fungus has not been known, to date, to produce a fungible product from biomass. Second, the lignin degradation capacity in oleaginous fungi could enable a highly consolidated platform for biomass conversion, where chemiphysical pretreatment might not be necessary. The combination of lignin modification with fungal fermentation has already reached a similar performance in terms of lipid yield (Fig. 3). Such a capacity can be further enhanced by genetic modification or condition optimization to achieve a more consolidated process. However, it should be pointed out that a limitation of FR3 fermentation is the relatively weak cellulose and hemicellulose degradation capacity. Third, the strong lignin degradation capacity also indicated that it is possible for FR3 to be utilized for the conversion of lignin. A fungusbased lipid production platform is often robust and more amenable to small to mid-size operations. The lignin utilization capacity and the potential for consolidated processing will enable these strengths. The utilization of lignin modification mutants may further enhance the advantages. Further engineering of FR3 and optimization of fermentation conditions are necessary to enable such platforms.

\section{Conclusions}

In summary, we have revealed that the oleaginous fungi C. echinulata FR3 can accumulate high level of lipid by degrading all the components of the plant cell wall including lignin, cellulose, and hemicellulose. The biomass composition analysis and NMR-based structure analysis indicated that C. echinulata FR3 degraded lignin with a higher efficiency than cellulose/hemicellulose. Further transcriptomics studies and 
biochemical studies revealed that lignocellulose degradation in C. echinulata FR3 involved both extracellular lignocellulolytic enzymes and Fenton-system-mediated hydroxyl radicals. In addition, lignin modification in plant feedstock could improve the lignocellulose-to-lipid conversion efficiency by C. echinulata FR3. In the extreme case, the lignin-modified sorghum feedstock could be used for $C$. echinulata FR3 fermentation to yield a similar amount of lipid as compared to pretreated wildtype plants. The study thus suggested that combining feedstock lignin modification with oleaginous fungal fermentation might deliver a cost-efficient bioconversion platform without chemical pretreatment. Considering the unique and strong lignin degradation capacity, the oleaginous fungus C. echinulata FR3 could serve as the base strain for further genetic engineering to achieve a sustainable and economical lignocellulose-to-biodiesel platform.

\section{Acknowledgements}

The work in China was supported by a grant from the National High-tech Research and Development Program of China (grant no. 2011AA100904). The work in the United States was supported by the U.S. DOE (Department of Energy) EERE (Energy Efficiency and Renewable Energy) BETO (Bioenergy Technology Office) (grant no. DE-EE0006112) to JSY and BY. The research was also supported by Texas A\&M Agrilife Research's biofuel initiative to JSY.

\section{References}

1 L. R. Lynd, M. S. Laser, D. Bransby, B. E. Dale, B. Davison, R. Hamilton, M. Himmel, M. Keller, J. D. McMillan, J. Sheehan and C. E. Wyman, Nat. Biotechnol., 2008, 26, 169-172.

2 B. Yang and C. E. Wyman, Biotechnol. Bioeng., 2004, 86, 88-95.

3 S.-Y. Ding, Y.-S. Liu, Y. Zeng, M. E. Himmel, J. O. Baker and E. A. Bayer, Science, 2012, 338, 1055-1060.

4 F. Chen and R. A. Dixon, Nat. Biotechnol., 2007, 25, 759761.

5 J. S. Yuan, K. H. Tiller, H. Al-Ahmad, N. R. Stewart and C. N. Stewart, Jr., Trends Plant Sci., 2008, 13, 421-429.

6 R. Franke, C. M. McMichael, K. Meyer, A. M. Shirley, J. C. Cusumano and C. Chapple, Plant J., 2000, 22, 223234.

7 F. Chen, M. S. Srinivasa Reddy, S. Temple, L. Jackson, G. Shadle and R. A. Dixon, Plant J., 2006, 48, 113-124.

8 C. Fu, J. R. Mielenz, X. Xiao, Y. Ge, C. Y. Hamilton, M. Rodriguez, Jr., F. Chen, M. Foston, A. Ragauskas, J. Bouton, R. A. Dixon and Z. Y. Wang, Proc. Natl. Acad. Sci. USA, 2011, 108, 3803-3808.

9 A. Saballos, W. Vermerris, L. Rivera and G. Ejeta, BioEnergy Res., 2008, 1, 193-204.
10 X. Li, J. K. Weng and C. Chapple, Plant J., 2008, 54, 569581.

11 K. S. Porter, J. D. Axtell, V. L. Lechtenberg and V. F. Colenbrander, Crop Sci., 1978, 18, 205-208.

12 Z. Xin, M. L. Wang, N. A. Barkley, G. Burow, C. Franks, G. Pederson and J. Burke, BMC Plant Biol., 2008, 8, 103.

13 N. A. Palmer, S. E. Sattler, A. J. Saathoff, D. Funnell, J. F. Pedersen and G. Sarath, Planta, 2008, 229, 115-127.

14 B. S. Dien, G. Sarath, J. F. Pedersen, S. E. Sattler, H. Chen, D. L. Funnell-Harris, N. N. Nichols and M. A. Cotta, BioEnergy Res., 2009, 2, 153-164.

15 J. Zeng, Y. Zheng, X. Yu, L. Yu, D. Gao and S. Chen, Bioresour. Technol., 2013, 128, 385-391.

16 H. Wei, W. Wang, J. M. Yarbrough, J. O. Baker, L. Laurens, S. Van Wychen, X. Chen, L. E. Taylor, II, Q. Xu, M. E. Himmel and M. Zhang, PLoS One, 2013, 8, e71068.

17 M. Kosa and A. J. Ragauskas, Green Chem., 2013, 15, 20702074.

18 C. Hu, X. Zhao, J. Zhao, S. Wu and Z. K. Zhao, Bioresour. Technol., 2009, 100, 4843-4847.

19 T. J. Cutright, Int. Biodeterior. Biodegrad., 1995, 35, 397408.

20 D. Müncnerová and J. Augustin, Bioresour. Technol., 1994, 48, 97-106.

21 J. F. Pedersen, J. J. Toy, D. L. Funnell, S. E. Sattler, A. L. Oliver and R. A. Grant, J. Plant Res., 2008, 2, 258-262.

22 W. Du, H. Yu, L. Song, J. Zhang, C. Weng, F. Ma and X. Zhang, Biotechnol. Biofuels, 2011, 4, 1754-6834.

23 S. Xie, S. Sun, S. Y. Dai and J. S. Yuan, Algal Res., 2013, 2, 28-33.

24 A. Sluiter, B. Hames, R. Ruiz, C. Scarlata, J. Sluiter, D. Templeton and D. Crocker, 2008, http://www.nrel.gov/ biomass/pdfs/42618.pdf.

25 M. G. Grabherr, B. J. Haas, M. Yassour, J. Z. Levin, D. A. Thompson, I. Amit, X. Adiconis, L. Fan, R. Raychowdhury, Q. Zeng, Z. Chen, E. Mauceli, N. Hacohen, A. Gnirke, N. Rhind, F. di Palma, B. W. Birren, C. Nusbaum, K. Lindblad-Toh, N. Friedman and A. Regev, Nat. Biotechnol., 2011, 29, 644-652.

26 W. Li and A. Godzik, Bioinformatics, 2006, 22, 1658-1659.

27 A. Dereeper, V. Guignon, G. Blanc, S. Audic, S. Buffet, F. Chevenet, J. F. Dufayard, S. Guindon, V. Lefort, M. Lescot, J. M. Claverie and O. Gascuel, Nucleic Acids Res., 2008, 36, 19.

28 S. Guindon, J. F. Dufayard, V. Lefort, M. Anisimova, W. Hordijk and O. Gascuel, Syst. Biol., 2010, 59, 307-321.

29 B. Dien, G. Sarath, J. Pedersen, S. Sattler, H. Chen, D. Funnell-Harris, N. Nichols and M. Cotta, BioEnergy Res., 2009, 2, 153-164.

30 G. J. Leary, K. R. Morgan, R. H. Newman, B. Samuelsson and U. Westermark, in $h f s g, 1986$, vol. 40, p. 221.

31 X. F. Sun, R. Sun, P. Fowler and M. S. Baird, J. Agric. Food Chem., 2005, 53, 860-870.

32 G. Gilardi, L. Abis and A. E. G. Cass, Enzyme Microb. Technol., 1995, 17, 268-275.

33 D. Singh, J. Zeng, D. D. Laskar, L. Deobald, W. C. Hiscox and S. Chen, Biomass Bioenergy, 2011, 35, 1030-1040. 
34 J. Zhang, Z. Zhu, X. Wang, N. Wang, W. Wang and J. Bao, Biotechnol. Biofuels, 2010, 3, 1754-6834.

35 S. I. Mussatto and I. C. Roberto, Bioresour. Technol., 2004, 93, 1-10.

36 D. Floudas, M. Binder, R. Riley, K. Barry, R. A. Blanchette, B. Henrissat, A. T. Martínez, R. Otillar, J. W. Spatafora, J. S. Yadav, A. Aerts, I. Benoit, A. Boyd, A. Carlson, A. Copeland, P. M. Coutinho, R. P. de Vries, P. Ferreira, K. Findley, B. Foster, J. Gaskell, D. Glotzer, P. Górecki, J. Heitman, C. Hesse, C. Hori, K. Igarashi, J. A. Jurgens, N. Kallen, P. Kersten, A. Kohler, U. Kües, T. K. A. Kumar, A. Kuo, K. LaButti, L. F. Larrondo, E. Lindquist, A. Ling, V. Lombard, S. Lucas, T. Lundell, R. Martin, D. J. McLaughlin, I. Morgenstern, E. Morin, C. Murat, L. G. Nagy, M. Nolan, R. A. Ohm, A. Patyshakuliyeva, A. Rokas, F. J. Ruiz-Dueñas, G. Sabat, A. Salamov, M. Samejima, J. Schmutz, J. C. Slot, F. St. John, J. Stenlid, H. Sun, S. Sun, K. Syed, A. Tsang, A. Wiebenga, D. Young, A. Pisabarro, D. C. Eastwood, F. Martin, D. Cullen, I. V. Grigoriev and D. S. Hibbett, Science, 2012, 336, 17151719.

37 C. Liers, T. Arnstadt, R. Ullrich and M. Hofrichter, FEMS Microbiol. Ecol., 2011, 78, 91-102.

38 M. J. Pecyna, R. Ullrich, B. Bittner, A. Clemens, K. Scheibner, R. Schubert and M. Hofrichter, Appl. Microbiol. Biotechnol., 2009, 84, 885-897.

39 M. Kinne, M. Poraj-Kobielska, S. A. Ralph, R. Ullrich, M. Hofrichter and K. E. Hammel, J. Biol. Chem., 2009, 284, 29343-29349.

40 P. Kersten and D. Cullen, Fungal Genet. Biol., 2007, 44, 77-87.

41 A. Vanden Wymelenberg, G. Sabat, M. Mozuch, P. J. Kersten, D. Cullen and R. A. Blanchette, Appl. Environ. Microbiol., 2006, 72, 4871-4877.

42 C. O. Rohr, L. N. Levin, A. N. Mentaberry and S. A. Wirth, PLoS One, 2013, 8, e81033.

43 A. Vanden Wymelenberg, J. Gaskell, M. Mozuch, G. Sabat, J. Ralph, O. Skyba, S. D. Mansfield, R. A. Blanchette, D. Martinez, I. Grigoriev, P. J. Kersten and D. Cullen, Appl. Environ. Microbiol., 2010, 76, 3599-3610.

44 D. Martinez, J. Challacombe, I. Morgenstern, D. Hibbett, M. Schmoll, C. P. Kubicek, P. Ferreira, F. J. Ruiz-Duenas, A. T. Martinez, P. Kersten, K. E. Hammel, A. Vanden
Wymelenberg, J. Gaskell, E. Lindquist, G. Sabat, S. S. Bondurant, L. F. Larrondo, P. Canessa, R. Vicuna, J. Yadav, H. Doddapaneni, V. Subramanian, A. G. Pisabarro, J. L. Lavin, J. A. Oguiza, E. Master, B. Henrissat, P. M. Coutinho, P. Harris, J. K. Magnuson, S. E. Baker, K. Bruno, W. Kenealy, P. J. Hoegger, U. Kues, P. Ramaiya, S. Lucas, A. Salamov, H. Shapiro, H. Tu, C. L. Chee, M. Misra, G. Xie, S. Teter, D. Yaver, T. James, M. Mokrejs, M. Pospisek, I. V. Grigoriev, T. Brettin, D. Rokhsar, R. Berka and D. Cullen, Proc. Natl. Acad. Sci. U. S. A., 2009, 106, 1954-1959.

45 C. Hori, J. Gaskell, K. Igarashi, M. Samejima, D. S. Hibbett,

B. Henrissat and D. Cullen, Mycologia, 2013, 105, 14121427.

46 F. Guillen, C. Munoz, V. Gomez-Toribio, A. T. Martinez and M. Jesus Martinez, Appl. Environ. Microbiol., 2000, 66, 170175.

47 J. Jensen, A. Kenneth, Z. C. Ryan, A. Vanden Wymelenberg, D. Cullen and K. E. Hammel, Appl. Environ. Microbiol., 2002, 68, 2699-2703.

48 S. Xie, R. Syrenne, S. Sun and J. S. Yuan, Curr. Opin. Biotechnol., 2014, 27, 195-203.

49 D. C. Eastwood, D. Floudas, M. Binder, A. Majcherczyk, P. Schneider, A. Aerts, F. O. Asiegbu, S. E. Baker, K. Barry, M. Bendiksby, M. Blumentritt, P. M. Coutinho, D. Cullen, R. P. de Vries, A. Gathman, B. Goodell, B. Henrissat, K. Ihrmark, H. Kauserud, A. Kohler, K. LaButti, A. Lapidus, J. L. Lavin, Y.-H. Lee, E. Lindquist, W. Lilly, S. Lucas, E. Morin, C. Murat, J. A. Oguiza, J. Park, A. G. Pisabarro, R. Riley, A. Rosling, A. Salamov, O. Schmidt, J. Schmutz, I. Skrede, J. Stenlid, A. Wiebenga, X. Xie, U. Kües, D. S. Hibbett, D. Hoffmeister, N. Högberg, F. Martin, I. V. Grigoriev and S. C. Watkinson, Science, 2011, 333, 762765.

50 G. Fuchs, M. Boll and J. Heider, Nat. Rev. Microbiol., 2011, 9, 803-816.

51 D. Martinez, L. F. Larrondo, N. Putnam, M. D. Gelpke, K. Huang, J. Chapman, K. G. Helfenbein, P. Ramaiya, J. C. Detter, F. Larimer, P. M. Coutinho, B. Henrissat, R. Berka, D. Cullen and D. Rokhsar, Nat. Biotechnol., 2004, 22, 695-700.

52 H. Doddapaneni, V. Subramanian, B. Fu and D. Cullen, Fungal Genet. Biol., 2013, 55, 22-31. 


\section{Supplementary data}

2 Table S1. Chemical composition of sorghum stover.

3 Table S2. ${ }^{13} \mathrm{C}$ chemical shift assignments in solid state CP-MAS analysis.

4 Figure S1. Released reducing sugar of untreated wild-type (WT), bmr6 mutant (b6), bmr12

5 mutant (b12) and bmr6/bmr12 double mutant (b6b12) sorghum stover after 48h hydrolysis with

6 different cellulase loadings (3.0, 4.5 and 6.0 FPU/g sorghum) at $28{ }^{\circ} \mathrm{C}$.

7 Figure S2. Reducing sugar concentration of the cultivation supernatant during the conversion of 8 wild type (solid square), bmr6 mutant (solid triangle), bmr12 mutant (open square) and 9 bmr6/bmr12 double mutant (open triangle) sorghum biomass by C. echinulata FR3.

10 Figure S3. Solid state ${ }^{13} \mathrm{C}$ CPMAS NMR analysis of control and fungus-treated sorghum 11 biomass. (A) wild-type sorghum without fungal conversion; (B) sample 1 of wild-type sorghum 12 after 6 days of fungal conversion by C. echinulata FR3; (C) sample 2 of wild-type sorghum after 136 days of fungal conversion by $C$. echinulata FR3; (D) sample 3 of wild-type sorghum after 6 14 days of fungal conversion by $C$. echinulata FR3; (E) bmr6/bmr12double mutant sorghum without fungal conversion; (F) sample 1 of bmr6/bmrl2double mutant sorghum after 6 days of fungal conversion by $C$. echinulata FR3; (G) sample 2 of bmr6/bmr12double mutant sorghum

17 after 6 days of fungal conversion by $C$. echinulata FR3; and $(\mathrm{H})$ sample 3 of bmr6/bmr12 double mutant sorghum after 6 days of fungal conversion by C. echinulata FR3. Figure S4. Comparison of the expanded ${ }^{13} \mathrm{C}$ CPMAS NMR region of fungus-conversed sorghum by C. echinulata FR3 and their corresponding controls. (A) aromatic resonances, (B) carbohydrate carbon resonances. (black) wild-type sorghum without fungal conversion; (red) sample 1 of wild-type sorghum after 6 days of fungal conversion by C. echinulata FR3; (blue) sample 2 of wild-type sorghum after 6 days of fungal conversion by C. echinulata FR3; (green) sample 3 of wild-type sorghum after 6 days of fungal conversion by $C$. echinulata FR3; (magenta) bmr6/bmr12 double mutant sorghum without fungal conversion; (lite blue) sample 1 of bmr6/bmr12 double mutant sorghum after 6 days of fungal conversion by C. echinulata FR3;

27 (orange) sample 2 of bmr6/bmr12 double mutant sorghum after 6 days of fungal conversion by $C$. echinulata FR3; and (olive) sample 3 of bmr6/bmr12 double mutant sorghum after 6 days of fungal conversion by C. echinulata FR3. 
30 Figure S5. GC/MS analysis of lipid profile of C. echinulata FR3 after growing 6 days on dilute 31 acid pretreated wild-type sorghum and un-pretreated bmr6/bmrl2 double mutant sorghum 32 biomass.

33 Figure S6. Functional analysis of lignocellulose degradation gene categories based on CAZy 34 database. (A). Distribution of glycoside hydrolases (GH), glycosyl transferases (GT), 35 polysaccharide lyases (PL), carbohydrate esterases (CE) and auxiliary activities (AA) among the 36 CAZy orthologous genes. (B). Distribution of each GH family among the putative glycoside 37 hydrolases genes.

38 Figure S7. Phylogenetic analysis of CeFR1943 laccase-like multiple copper oxidase with the 39 other 35 published laccases from 30 different species including fungus (ascomycetes and 40 basidiomycetes) and plants. 
42

\begin{tabular}{lllll}
\hline Genotype & Cellulose (\%) & Hemicellulose (\%) & Lignin (\%) & Ash (\%) \\
\hline Wild type & 24.71 & 19.00 & 17.28 & 4.42 \\
bmr6 mutant & 25.31 & 19.85 & 16.49 & 5.20 \\
bmr12 mutant & 24.60 & 19.30 & 16.72 & 5.52 \\
bmr6/bmr12 & 24.10 & 18.45 & 15.50 & 6.23 \\
double mutant & & & \\
\hline
\end{tabular}

43

44

Table S1

45 


\begin{tabular}{ll}
\hline $\boldsymbol{\delta}_{\mathbf{C}}(\mathrm{ppm})$ & Assignment \\
\hline 198 & Aromatic carbonyl (-CO-) resonances \\
$185-175$ & Aliphatic carbonyl/carboxyl (-COOR, -COO-) resonances \\
$160-200$ & Carbonyl and carboxyl carbon resonances \\
$175-165$ & Aromatic carboxyl (-COO-) resonances \\
$155-160$ & C4 resonances in $p$-coumarate ester/p-hydroxyphenyl unit \\
$100-162$ & Aromatic ring resonances (H,G,S) \\
$149-152$ & C3,C5 in etherified S- and C3 in etherified G-unit \\
$147-149$ & C3, C5 in non-etherified S- and C3 in non-etherified G-unit. \\
$136-140$ & C1 resonances in etherified H,G and S-unit \\
$132-134$ & C1 resonances in non-etherified H,G and S-unit \\
$103-130$ & C2, C6 in S-/C3,C5 in H-/C5 in G-unit \\
$60-100$ & Carbohydrate carbon resonances \\
$52-55$ & Methoxyl in S- and G-unit \\
$40-20$ & Aliphatic carbon resonances of fatty acids \& triglycerides \\
\hline
\end{tabular}




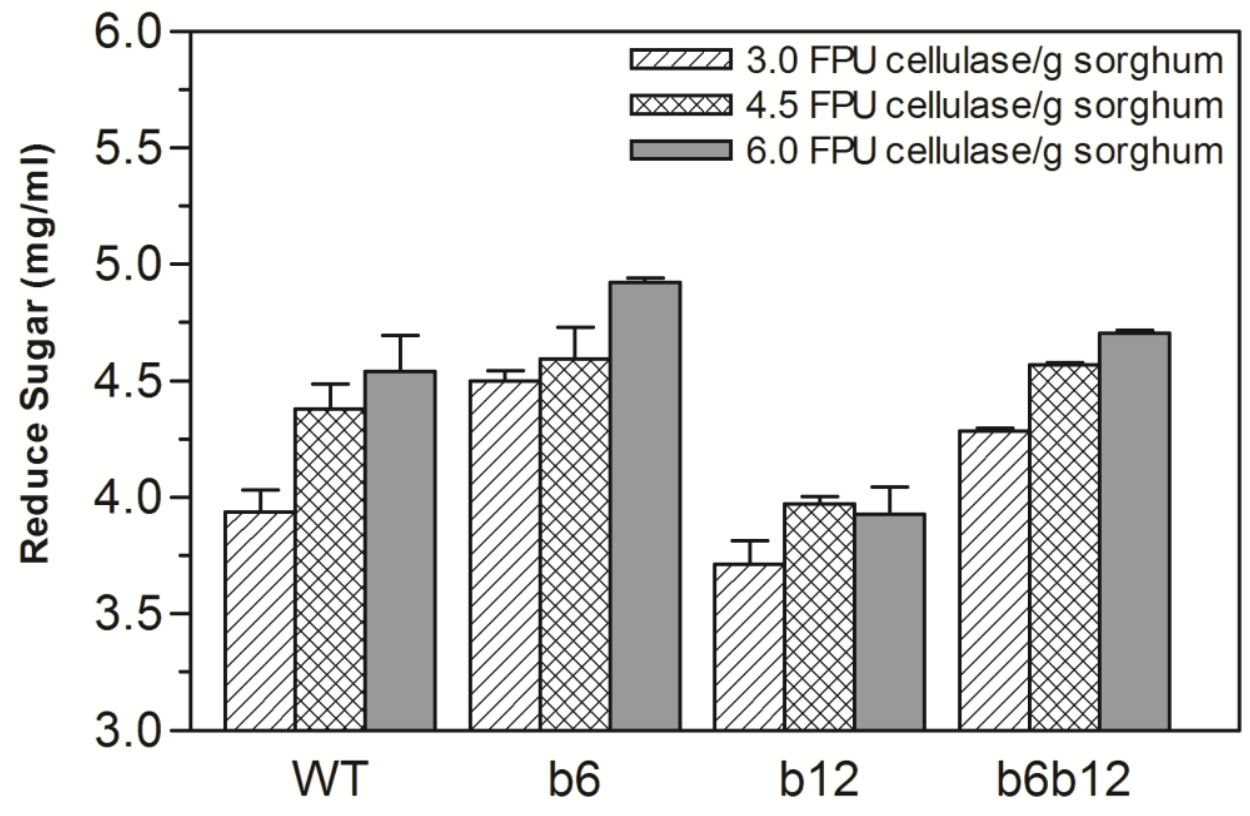

49

50

Figure S1

51 


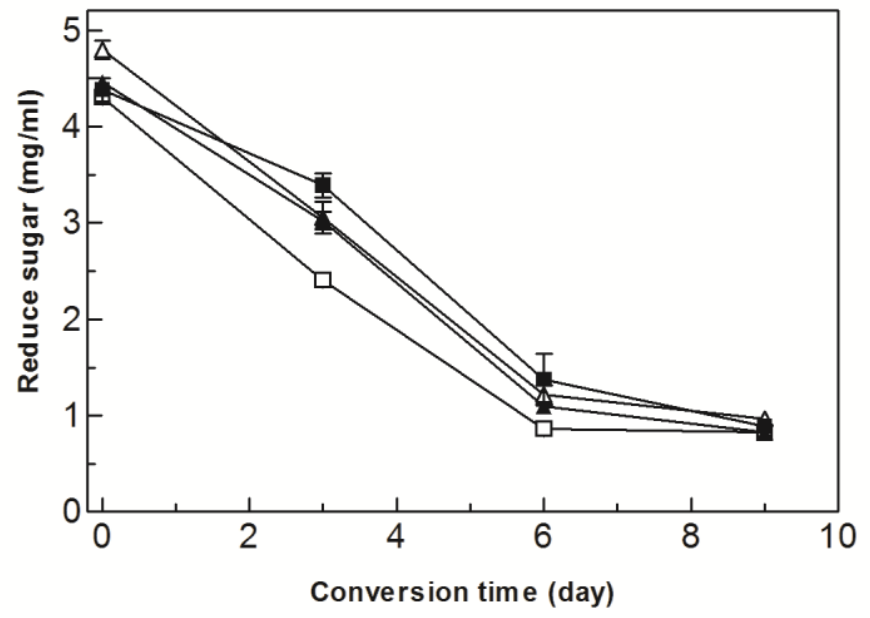

52

Figure S2 


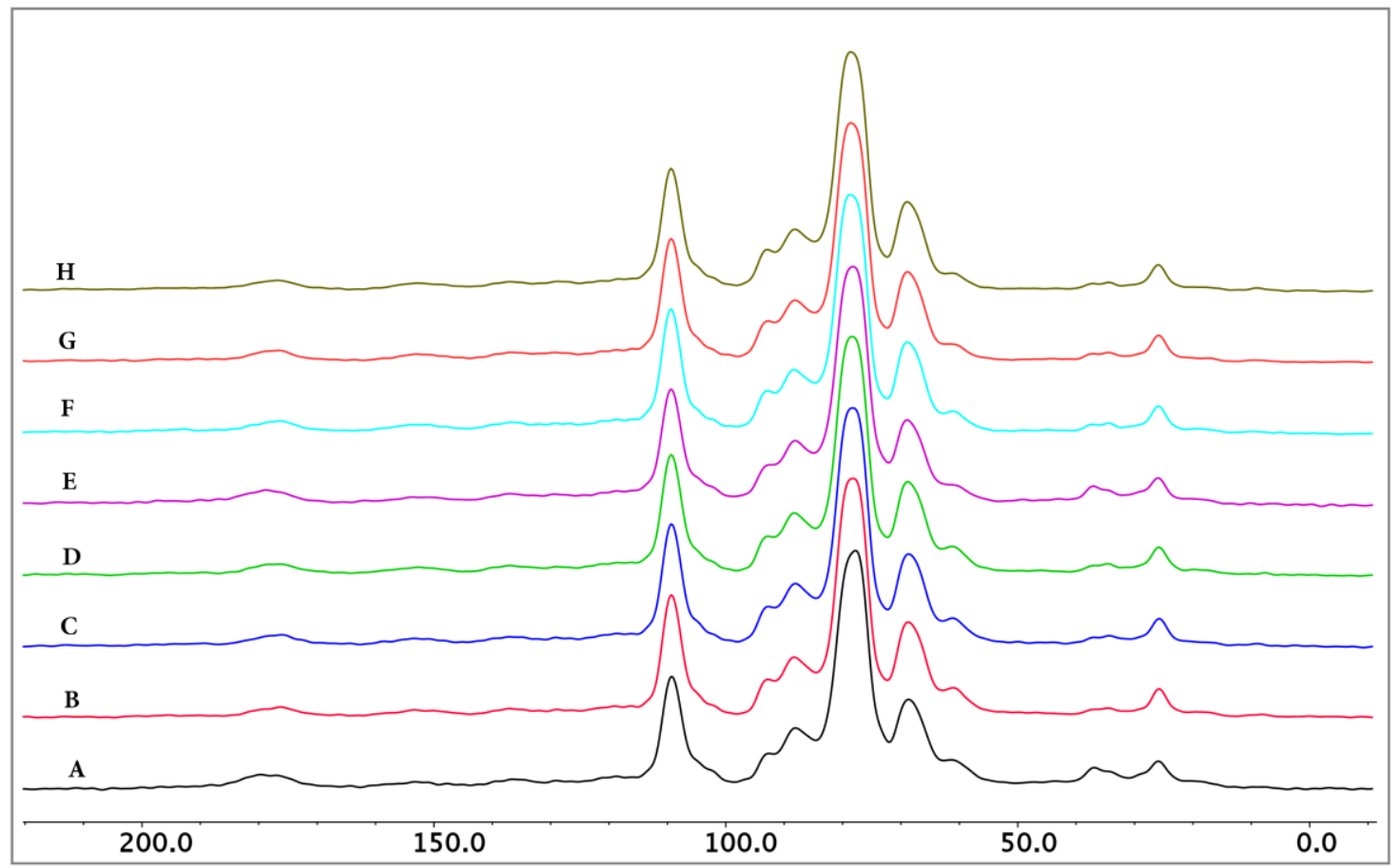

55

56

Figure S3

57 
A
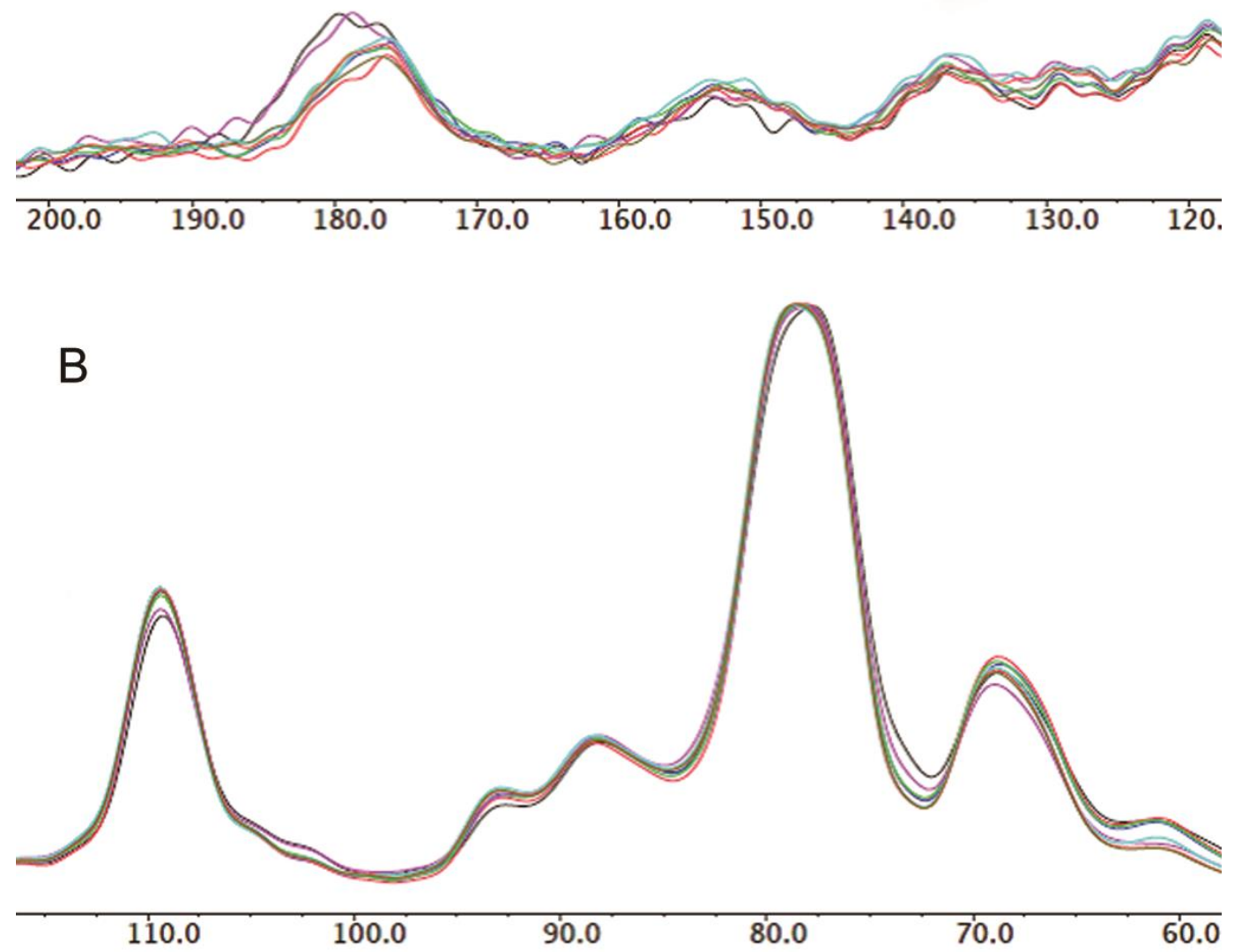

59

Figure S4

60 


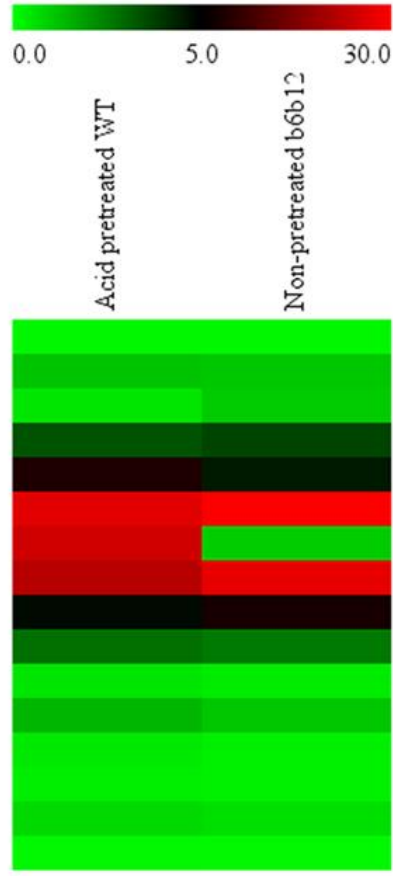

Methyl tetradecanoate

9-Hexadecenoic acid, methyl ester, (Z)-

Hexadecenoic acid, methyl ester, $(Z)$ -

Pentadecanoic acid, 14-methyl-, methyl ester

7,10-Octadecatrienoic acid, methyl ester

6,9,12-Octadecatrienoic acid, methyl ester

9,12-Octadecenoic acid, methyl ester, (E)-

9-Octadecenoic acid, methyl ester

9-Octadecenoic acid, methyl ester

Octadecenoic acid, methyl ester

9,12,15-Octadecatrienoic acid, methyl ester, $(Z, Z, Z)$ -

11-Eicosenoic acid, methyl ester

Eicosanoic acid, methyl ester

Docosanoic acid, methyl ester

Tetracosanoic acid, methyl ester

Hexacosanoic acid, methyl ester 


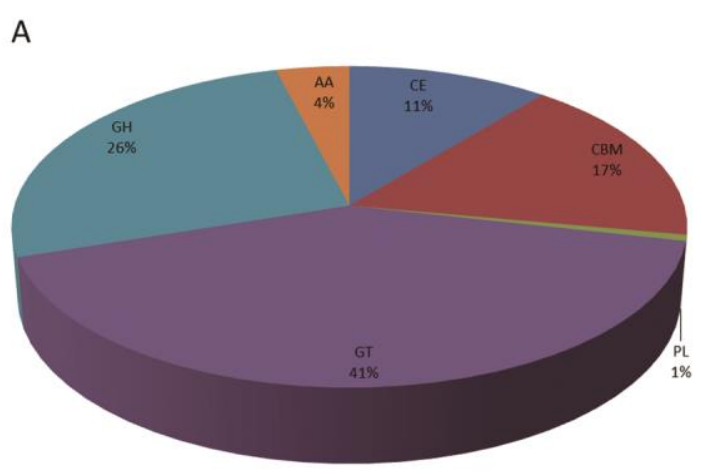

64

65

66

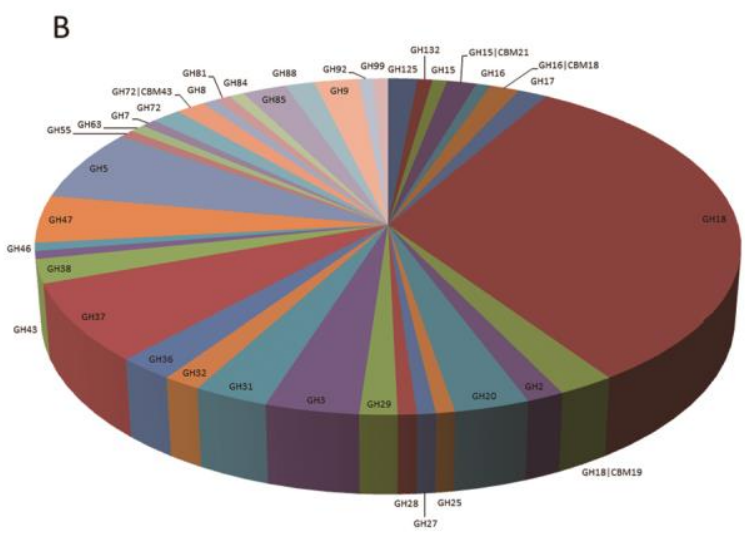

Figure S6 


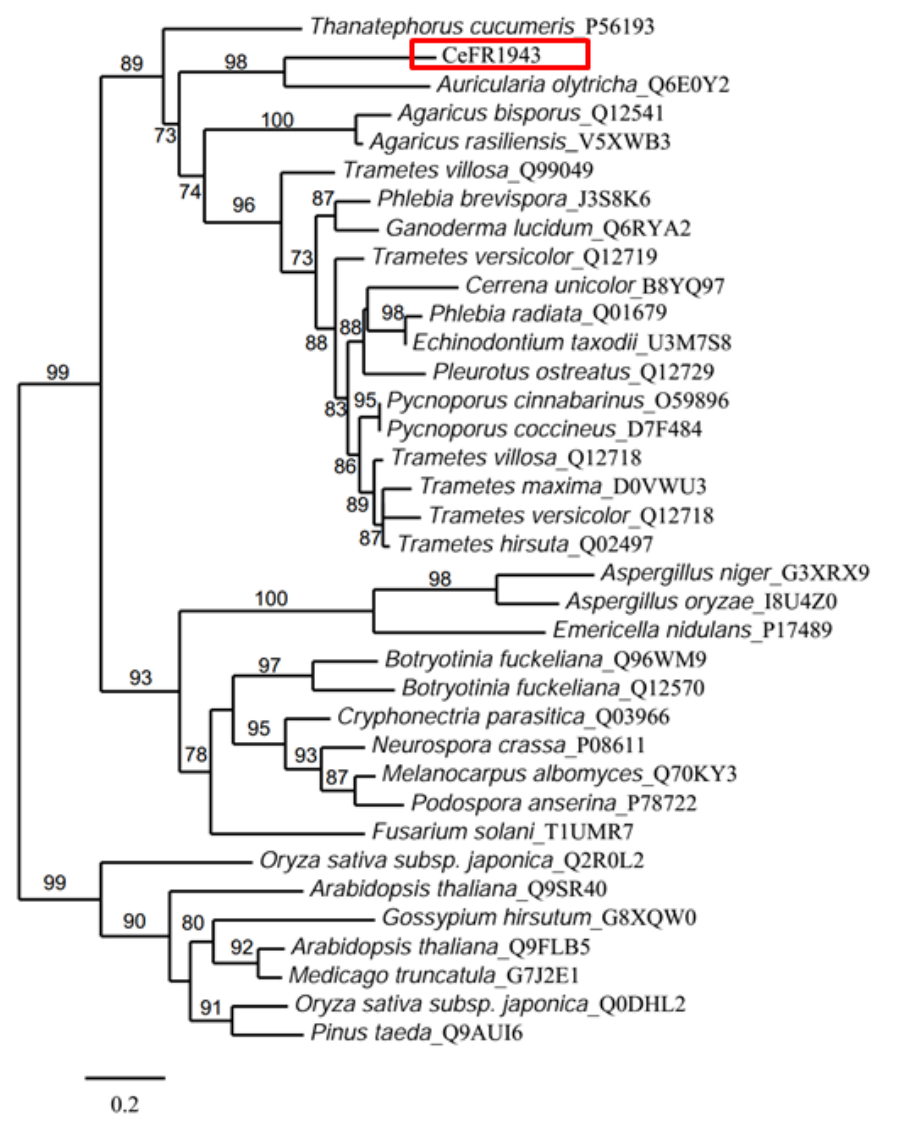

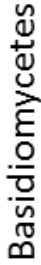

さ્さ

$\frac{\vec{c}}{\frac{\pi}{\alpha}}$

67

Figure S7

69

70 